\title{
Exosomes in the Pathogenic Protozoan Trypanosoma Cruzi
}

\author{
Wanderley de Souza ${ }^{1,2 *}$ and Emile Barrias ${ }^{3}$ \\ ${ }^{1}$ Instituto de Biofísica Carlos Chagas Filho, Universidade Federal do Rio de Janeiro, Brazil \\ ${ }^{2}$ Instituto Nacional de Ciência e Tecnologia em Biologia Estrutural e Bioimagens, Universidade Federal do Rio de Janeiro, Brazil \\ ${ }^{3}$ Instituto Nacional de Metrologia, Normalização, Qualidade e Tecnologia-Inmetro, Brazil
}

* Corresponding author: Wanderley de Souza, Instituto de Biofísica Carlos Chagas Filho and Núcleo de Biologia Estrutural e Bioimagens-CENABIO, Instituto Nacional de Ciência e Tecnologia em Biologia Estrutural e Bioimagens, Universidade Federal do Rio de Janeiro, Rio de Janeiro, Brazil, E-mail: wsouza@biof.ufrj.br

\begin{abstract}
Significant advances have recently occurred in the identification and characterization of different types of microvesicles released by eukaryotic cells into the extracellular space. Exosomes are one such type of these vesicles they originate from multivesicular bodies and have received much attention. Here, we review the available data on microvesicles secreted by the pathogenic protozoan Trypanosoma cruzi, which is the causative agent of Chagas disease and that still has a high prevalence in Latin America. The available data show that $T$. cruzi exosomes contain some key proteins and RNA molecules that contain genetic information and may interfere with transcription. The available information suggests that they play some role in the biology of $T$. cruzi, including its interaction with host cells, as well as on the pathogenesis of Chagas disease where inflammatory processes and host cell dead occurs as a consequence of parasites, factors released by the parasites, and molecules produced by different host cells that are part of the immunological response.
\end{abstract}

\section{Keywords}

Trypanosoma cruzi, Exosome, Microvesicles

\section{Introduction}

Basic knowledge on the organization of eukaryotic cells is established in many textbooks [1]. However, advances in the association of morphological with biochemical and molecular techniques have added new structures and organelles. Examples include exosomes and ectosomes. Although observed for many years, their role in cell-to-cell communication has only recently been understood. Recent data clearly shows that they are involved in cell-to-cell communication - a function usually considered occurring exclusively in gap junctions and in secretory processes where the mediators as ligands, chemical and transmitors are released in the vicinity where the receptors are located, e.g., neuron-to-neuron or neuron-to-muscle cells synapses.

Early studies carried out by Pan and Johnstone [2,3] using reticulocytes showed that inward budding of endosomal membranes resulted in the accumulation of intraluminal vesicles within large multivesicular bodies. Some of these bodies migrate to the periphery of the cell followed by fusion with the plasma membrane and release of the vesicles into the extracellular space. These are then designated exocytic multivesicular bodies and later designated as exosomes [4]. It was later shown that vesicles with different diameters (varying from 40 up to $1000 \mathrm{~nm}$ ) can be found in the extracellular space and designated by several names such as microvesicles, microparticles, etc. They are found around cells as well as in all biological fluids. Collectively they have been designated as "extracellular microvesicles" [5].

Several reports have shown that extracellular vesicles may be formed by different mechanisms initiated by the outward budding of the plasma membrane or from the endosomal system $[6,7]$. Some originate from membrane exfoliation, while others are shed as a final step in the process of capping the surface ligands - a process that occurs in free cells such as lymphocytes following ligand-receptor binding and mobility [8].

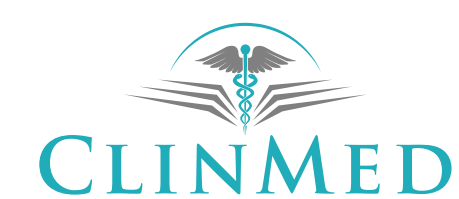

INTERNATIONAL LIBRARY
Citation: de Souza W, Barrias E (2017) Exosomes in the Pathogenic Protozoan Trypanosoma Cruzi. Int J Pathol Clin Res 3:054. doi.org/10.23937/2469-5807/1510054

Received: February 23, 2017: Accepted: May 20, 2017: Published: May 22, 2017

Copyright: (c) 2017 de Souza W, et al. This is an open-access article distributed under the terms of the Creative Commons Attribution License, which permits unrestricted use, distribution, and reproduction in any medium, provided the original author and source are credited. 


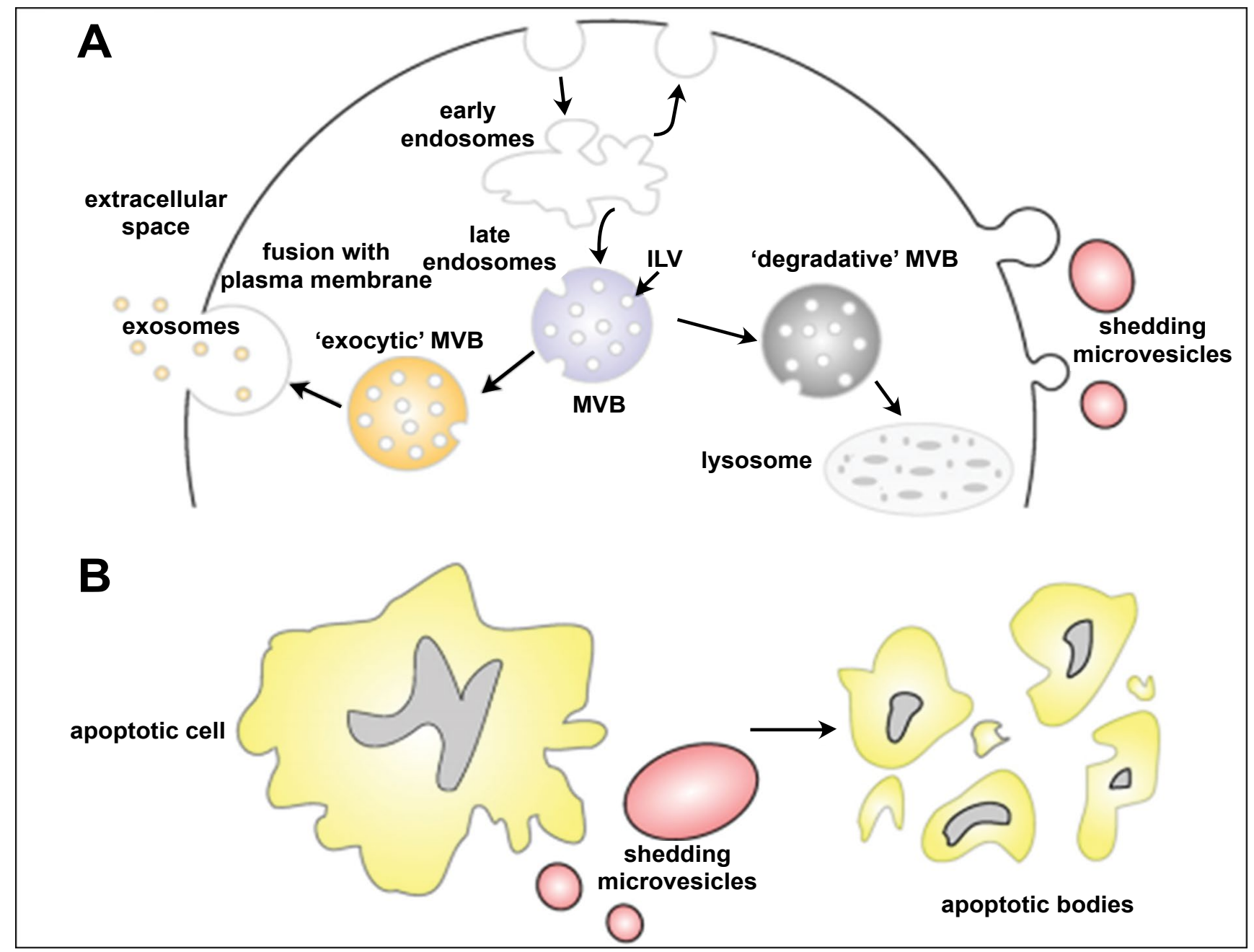

Figure 1: Schematic representation of the release of extracellular membranous microvesicles into the extracellular space. Adapted from Mathivanan, et al. [4].

On the other hand, vesicles are formed by the association of some macromolecular complexes with the inner face of the plasma membrane leading to budding of the membrane and release of vesicles outside the cell in a process resembling viral budding. These vesicles were designated as ectosomes [7]. Still others are released when cytoplasmic Multivesicular-Like Bodies (MVB) fuse with the plasma membrane and discharge their vesicular content into the extracellular space. This type of vesicles with a diameter of 40 to $100 \mathrm{~nm}$ is presently known as the exosome. Figure 1 shows a diagram that distinguishes these different processes [5].

Where are the exosomes found? Current data indicate that exosomes exist in a large number of cells, interstitial fluids, urine, milk, and even in snake venom [912]. Multivesicular Bodies (MVBs) are always involved and are an intermediate. A well-defined compartment is formed during invagination of the membrane lining the endosome. It is important to point out that in most cases, the MVB fuses with lysosomes and thus its cargo is degraded. In other situations however, the MVB fuses with the plasma membrane releasing the exosomes [13]. The fusion process may be triggered by calcium ionophores [14].
The protein and nucleic acids contents of the exosome have been studied in detail. Isolated extracellular vesicles have been analyzed using different techniques including mass spectrometry. Several proteins such as Rabs (Ras-related proteins in brain), annexins, adhesion molecules, cytoskeletal proteins and many others have been identified. Rab 27A and Rab 27B GTPases have been involved as exosome effector proteins [15-17]. More importantly, exosomes produced by mast cells contain about 1300 mRNAs and 121 microRNAs, but not DNA or rRNA $[18,19]$.

Once released into the extracellular space, the exosomes may bind to the surface of cells and fuse with it $[20,21]$ with subsequent opening of the vesicle inside the target cell or degradation of the exosome membrane [22]. This demonstrates a novel mode of communication between cells in addition to other well characterized structures such as synapsis and gap junctions. For instance, incubation of human cells with exosomes from mouse cells has led to the synthesis of mouse proteins from the mRNAs present in the exosomes. This indicates a process of horizontal gene transfer $[14,18,23]$.

Viruses, prions and other infectious materials can be transmitted from one cell to another via exosomes 


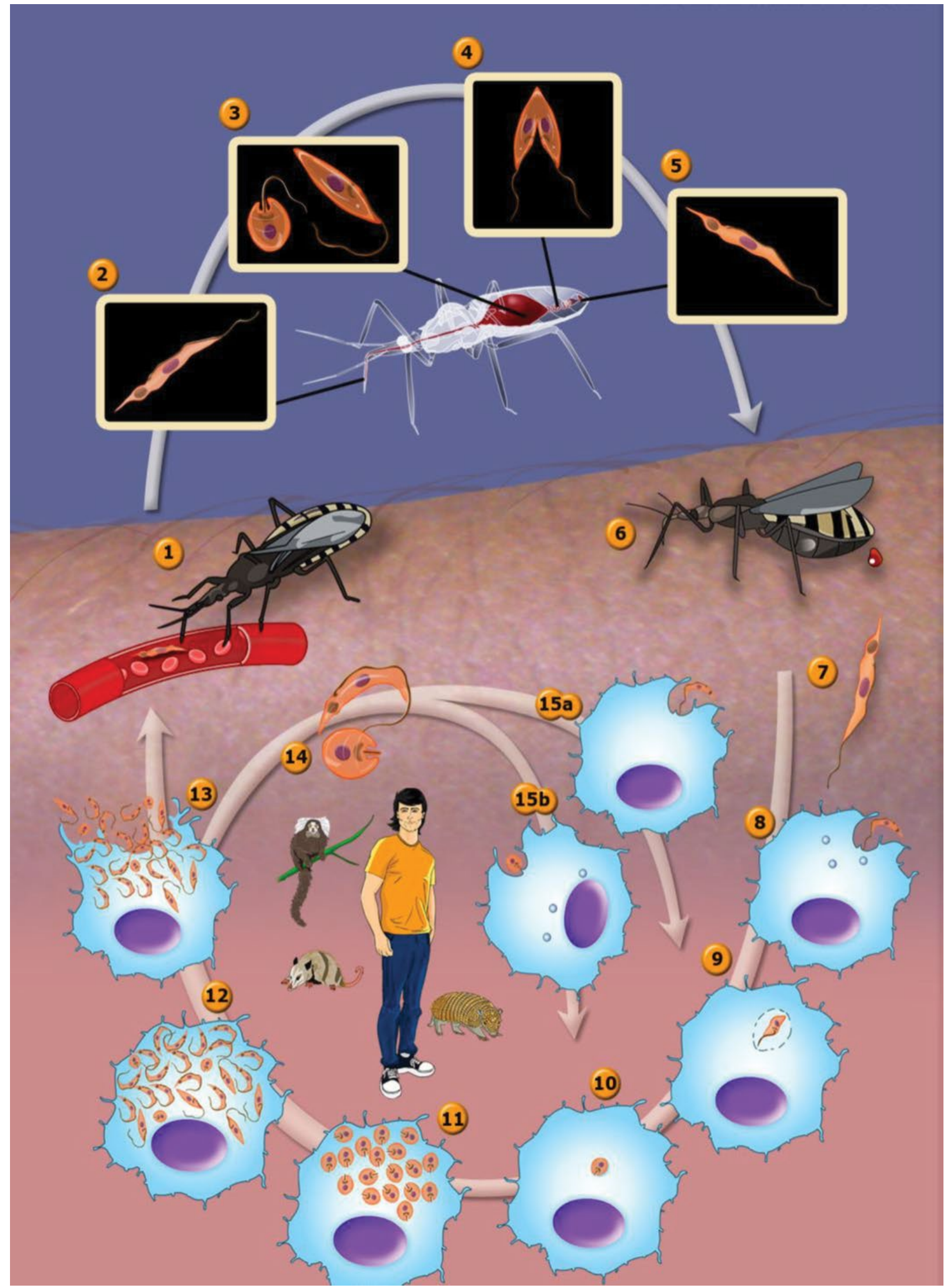

Figure 2: The life cycle of T. cruzi (1) The insect vector (female or male) bites a mammalian host and ingests trypomastigotes located in the blood; (2) Metacyclic trypomastigotes; (3) Trypomastigotes transform into epimastigotes and some spheromastigotes; (4) Epimastigotes multiply in the midgut; (5) Epimastigotes transform into metacyclic trypomastigotes in the hindgut; (6) The insect vector passes the metacyclic trypomastigotes in feces near a bite site after feeding on a mammalian host; (7) Metacyclic trypomastigotes form; (8) Metacyclic trypomastigote infects macrophages; (9) Metacyclic trypomastigote transforms into amastigote; (10) Amastigote is released from the parasitophorous vacuole; (11) Amastigotes multiply in the cytoplasm; (12) Amastigotes transform into trypomastigotes; (13) Trypomastigotes burst out of the cell; (14) Amastigotes and trypomastigotes form; (15) (a) Trypomastigotes and (b) Amastigotes infect macrophages. In the central portion of the figure, we added the most important animal reservoirs involved in the maintenance of the parasite in the domestic and peridomestic environment. After Teixeira, et al. [61]. 
[11,24-27]. They also act as vectors for mRNAs, miRNAs, virulence factors, and different types of mediators $[22,28]$. One good example of the role played by exosomes on the transfer of RNA is a recent study by Bukong and colleagues [29]. They showed that exosomes isolated from the sera of chronic Hepatitis C Virus (HCV)-infected patients or the supernatants of $\mathrm{HCV}$-infected cells contained HCV RNA and that they could mediate viral receptor-independent transmission of HCV to hepatocytes. These authors also showed that transmission of HCV was suppressed by some compounds, which inhibit proteins such as Heat Schock Protein (HSP90); vacuolar $\mathrm{H}^{+}$-ATPase significantly suppressed exosome-mediated HCV transmission to naïve cells.

The data indicate that virtually all cells can produce exosomes, but the role in parasites is less clear. Studies of Leishmania [30,31], Trypanosoma brucei [32,33] and Plasmodium [34] have clearly shown that these parasites also produce microvesicles that are released into the intercellular space or the bloodstream. Exosomes secreted by $P$. falciparum are directly related to the infection of red blood cells in a mechanism that seems to be important for the parasite's transmission to the insect vector [34]. In Leishmania sp., the exosome pathway is a non-conventional protein secretion mechanism because most exosome proteins do not contain a predicted signal peptide [30]. However, substantial work on exosome biogenesis, content and functions in several organisms, including Leishmania, has demonstrated that this pathway is far from unusual and is a major mechanism for systemic and microenvironmental immune manipulation [35]. More recently exosomes have been described in other pathogenic eukaryotic microorganisms but this is not the scope of this review $[36,37]$.

In this short review, we will focus only on the data available for Trypanosoma cruzi - the causative agent of Chagas disease that has a high prevalence in Latin America [38]. This protozoan presents a complex life cycle both in the invertebrate and vertebrate hosts where several developmental stages can be found. Figure 2 shows a general view of the life cycle which takes place in the invertebrate (triatomines) and vertebrate (mammals) hosts. Let us consider that the cycle starts with insects from the Reduvidae family sucking the blood of vertebrates infected with the parasite and containing trypomastigote forms which circulate in the bloodstream (known as bloodstream trypomastigotes). Once ingested together with the blood most of the trypomastigotes are lysed following interaction with bacteria existent in the insect stomach [39]. The surviving trypomastigotes transform a few days later either in spherical forms, known as spheromastigotes, or in epimastigote forms. The latter ones migrate to the intestine where they intensely divide and attach, through a specific interaction process where GPI-linked macromolecules exposed on the parasite surface are recognized by components of the intestinal cell surface and the perimicrovillar membranes [40]. At the most posterior regions of the intestine and at the rectum many epimastigotes detach from the intestinal surface and transform into trypomastigote forms which are then released together with the feces or with the urine [41]. These forms are also designated as metacyclic trypomastigotes which are highly infective for several mammal species, including the man. Usually the infection of mammals takes place through direct inoculation of these forms through the ocular mucosa or the lesioned skin following insect biting. More recently it has been shown that the forms are also infective through an oral route [41]. Once in the vertebrate host the metacyclic trypomastigotes invade cells found in the inoculation site (fibroblasts, macrophages, epithelial cells, etc) through several mechanisms (phagocytosis and induced endocytosis) and are then found within an endocytic vacuole known as the parasitophorous vacuole. A few hours after cell infection the long and thin trypomastigote form gradually became rounded and with a short flagellum, characteristics of the amastigote (also known as intracellular spheromastigote) form. At the same time the parasite secretes enzymes which help in the digestion of the membrane lining the parasitophorous vacuole $[42,43]$ so that the amastigote forms enter in direct contact with host cell organelles. Figure 3 shows schematically the various phases of the interaction of the protozoan with host cells.

Chagas' Disease (CD), recognized by the World Health Organization (WHO) as one of the world's 13 most neglected tropical diseases, continues to be a relevant social and economic problem in many American countries. Caused by the protozoan Trypanosoma cruzi, the overall illness prevalence is estimated to be approximately 16-18 million cases [39]. Among those infected, $30-40 \%$ will subsequently develop the chronic form of the disease. Chronic Chagas cardiomyopathy is the most serious outcome, leading to conduction abnormalities, cardiac failure and thromboembolism [39].

\section{Exosomes in the protozoan Trypanosoma cruzi}

Schmunis, et al. [44] used transmission electron microscopy to show vesicles shed from bloodstream trypomastigotes during capping of antigen-antibody complexes. Subsequently, Gonçalves, et al. [45] showed release of $20-80 \mathrm{~nm}$ vesicles resembling what is now known as exosomes along the entire extent of tissue culture-derived trypomastigotes. Vesicles with these dimensions can be observed by Scanning Electron Microscopy (SEM) at the infection moment (Figure 4). Immunocytochemical analysis showed that Tc85 (a member of the superfamily gp 85 trans-sialidase that is anchored to the plasma membrane via GPI-anchors) is localized in these vesicles. Injection of these vesicles into mice before infection with $T$. cruzi increased parasitaemia and led to exacerbation of heart lesions and inflammation [46]. All mice previously injected with the vesicles and 

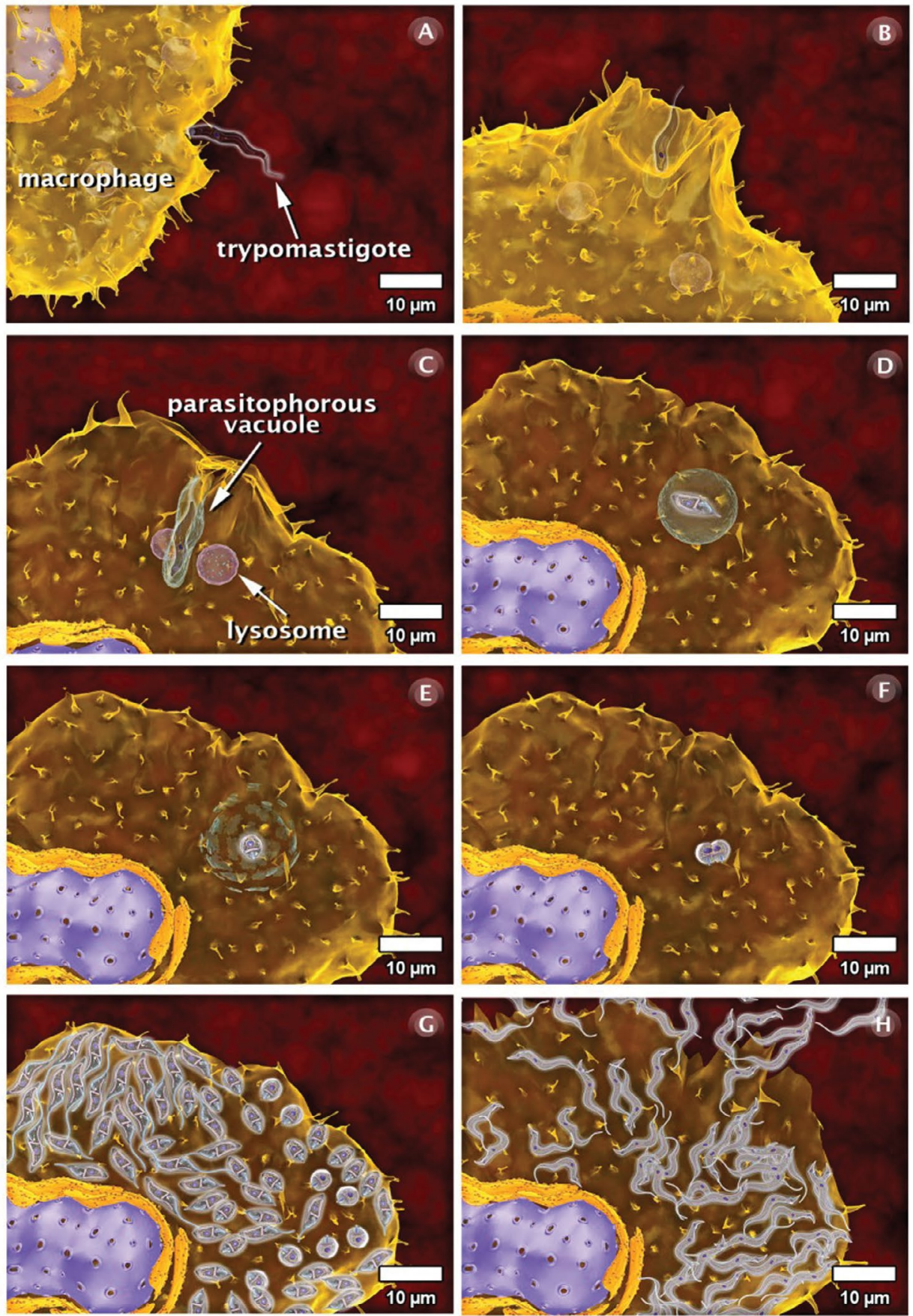

Figure 3: Interaction's phases of trypomastigote form of $T$. cruzi with vertebrate cells (A) Attachment of the trypomastigote form to the cell surface; (B) The process of internalization via phagocytosis begins with the formation of pseudopods and is followed by the recruitment and fusion of host cell lysosomes; (C) A parasitophorous vacuole is subsequently formed. The lysosomal content is released into the vacuole, and the parasite is not affected; (D) In the vacuole, the trypomastigote transforms into the amastigote form; (E) This transformation is accompanied by the digestion of the parasitophorous vacuole membrane; (F) The amastigote is released into the cytoplasm of the host cell and divide several times; (G) Following division, the amastigotes transform into trypomastigotes, which show intense and constant movement; $(\mathrm{H})$ The host cell bursts and the parasites reach the extracellular space and, subsequently, the bloodstream. These images were made based on micrographs of transmission electron microscopy and video microscopy. After Teixeira, et al. [61].

then infected with $T$. cruzi died after 20 days of infection whereas $20 \%$ of the untreated mice were still alive after 30 days thus indicating some partial protection. The mice previously injected with vesicles also displayed more severe heart pathology with predominance of TCD4+ lymphocytes and macrophages, but not of TCD8+. It was suggested that the vesicles led to an increase in tissue parasitism and inflammation due to stimulation of IL-4 and IL-10 synthesis. Subsequently it was shown that the vesicles contain ecto-phosphatases that could be inhibited by inhibitors such as sodium tartrate and sodium fluoride thus suggesting being acid phosphatases [47]. Inhibitors of phosphotyrosine phoshatase such as o-vanadate also inhibit enzyme activity.

Taking into consideration previous studies carried out to characterize the surface of $T$. cruzi it was suggested that the secreted phosphatases are biochemically related to those found on the parasite surface. The au- 


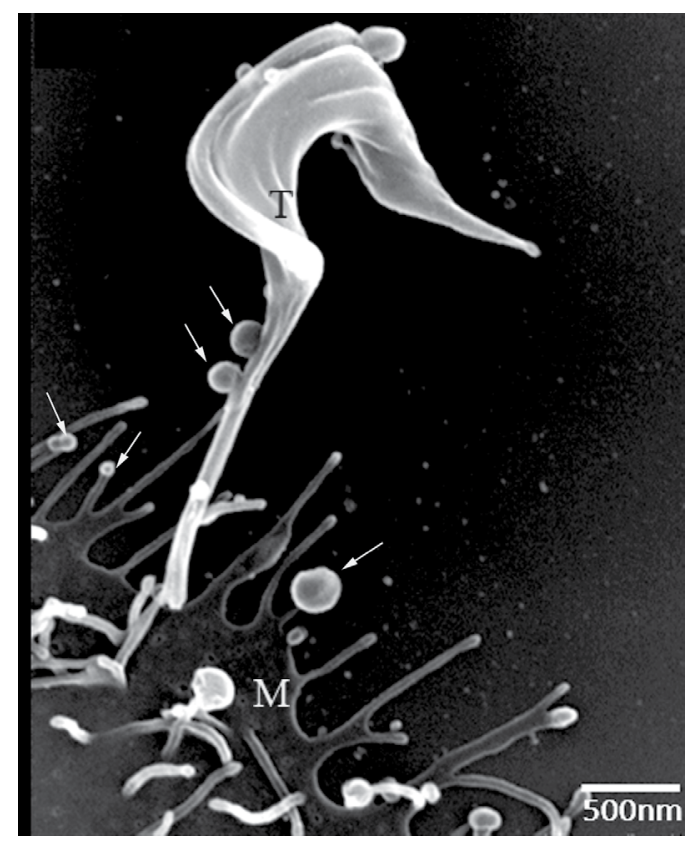

Figure 4: Scanning electron micrograph of interaction between trypomastigotes and host cell demonstrating the presence of shedding vesicles similar to exosomes. Asterisks: shedding vesicle; $\mathrm{T}$ : trypomastigote; M: mammalian cell.

thors also reported that these results varied according to the strain of $T$. cruzi used. Evidence was obtained showing that the phosphatases play some role on the process of adhesion of $T$. cruzi trypomastigotes to the host cell surface and its subsequent internalization [47].

More recently Garcia Silva, et al. [48] reported the presence of a homogeneous population of small RNAs derived from mature tRNAs that accounted for about $25 \%$ of the small RNA population in $T$. cruzi epimastigotes. The levels of these tRNA-derived Halves (tsRNAs) were more pronounced in parasites undergoing a process of nutritional stress that usually triggers differentiation into infective trypomastigotes. Interestingly, the tsRNAs were localized in a cytoplasmic organelle resembling the reservosomes [49]. This organelle is involved in the accumulation of both macromolecules ingested through the endocytic pathway as well as some proteases, especially cruzipain, that is synthesized by the parasite [50]. It was subsequently shown that tsRNAs were localized in organelles associated with clathrin and with the $T$. cruzi argonate protein TcPIWI-tryp that is morphologically characterized as reservosomes as well as in uncharacterized cytoplasmic vesicles and in Golgi-like vesicles [51]. It was also shown that $20-200 \mathrm{~nm}$ vesicles are released from the parasites into the extracellular medium from reservosome-like structures containing vesicles that touch the cytoplasmic face of the plasma membrane. This is especially true in epimastigotes submitted to nutrient starvation.

This may correspond to exosomes as well as other microvesicles such as ectosomes or shedded vesicles because the vesicle population is not homogenous. Bayer-Santos, et al. [52] reported the presence of two classes of vesicles: likely exosomes and ectosomes. The released vesicles carry small tRNAs and TcPIWI-tryp proteins, and they are internalized by other parasites suggesting that at least tRNA-derived small RNAs are delivered from one parasite to other parasites in the population.

It was also noted that the released vesicles stimulated the transformation of epimastigotes into trypomastigotes - a process known as metacyclogenesis [52]. The vesicles are also incorporated by mammalian cells susceptible to $T$. cruzi infection. Incorporation of the vesicles by electroporation into K562 cells that usually are not infected with $T$. cruzi render them susceptible to infection. Subsequently it was shown that incubation of mammalian cells with isolated vesicles from $T$. cruzi induces changes in gene expression as assessed by microarray assays. A large set of genes in HeLa cells were differentially expressed upon incorporation of $T$. cruzi-derived extracellular vesicles. This response mainly modified the host cell cytoskeleton, extracellular matrix, and immune responses pathways. The most abundant tRNA-derived small RNAs included in extracellular vesicles also modified some genes. These data suggest that microvesicles secreted by $T$. cruzi could affect parasite host cell interplay. It is now accepted that secreted exosomes and shed microvesicles/ectosomes are a means for the delivery of genetic information (e.g., miRNAs and mRNAs) and proteins between cells [19]. Interestingly, these exosomal mRNAs and microRNAs were completely functional in recipient cells and thus played pivotal roles in cell-to-cell communication [19]. Thus, it is possible to speculate that $T$. cruzi extracellular vesicles and their cargo could represent a route of intercellular communication delivering "molecular messages" to others cells aimed to induce coordinated responses to assure parasite survival through both the emergence of infective forms and the establishment of a cellular environment able to facilitate infection.

In this respect, it was recently reported that $T$. cruzi trypomastigotes invade 5 -fold as many susceptible cells when pre-incubated with purified parasite extracellular vesicles [53]. These results suggest that secreted vesicles from $T$. cruzi and their cargo could act as virulence factors by promoting metacyclogenesis and enhancing host cell susceptibility or both [53]. T. cruzi virulence factors include molecules expressed on the cell surface as well as those secreted or shed into the extracellular medium. Phosphatase activities modulate different aspects of $T$. cruzi infection, and Neves and colleagues [47] demonstrated the presence and activity of phosphatases in vesicles secreted by $\mathrm{Y}$ and CL-Brener (VF$\mathrm{CLB}$ ) trypomastigotes suggesting that exosomes are truly involved in $T$. cruzi invasion (Figure 5).

Cestari, et al. [54] reported that $T$. cruzi also induces release of exosomes from the host cells and that these vesicles bind to the parasites and protect them to complement-mediated lysis. Recently, Ramirez and 


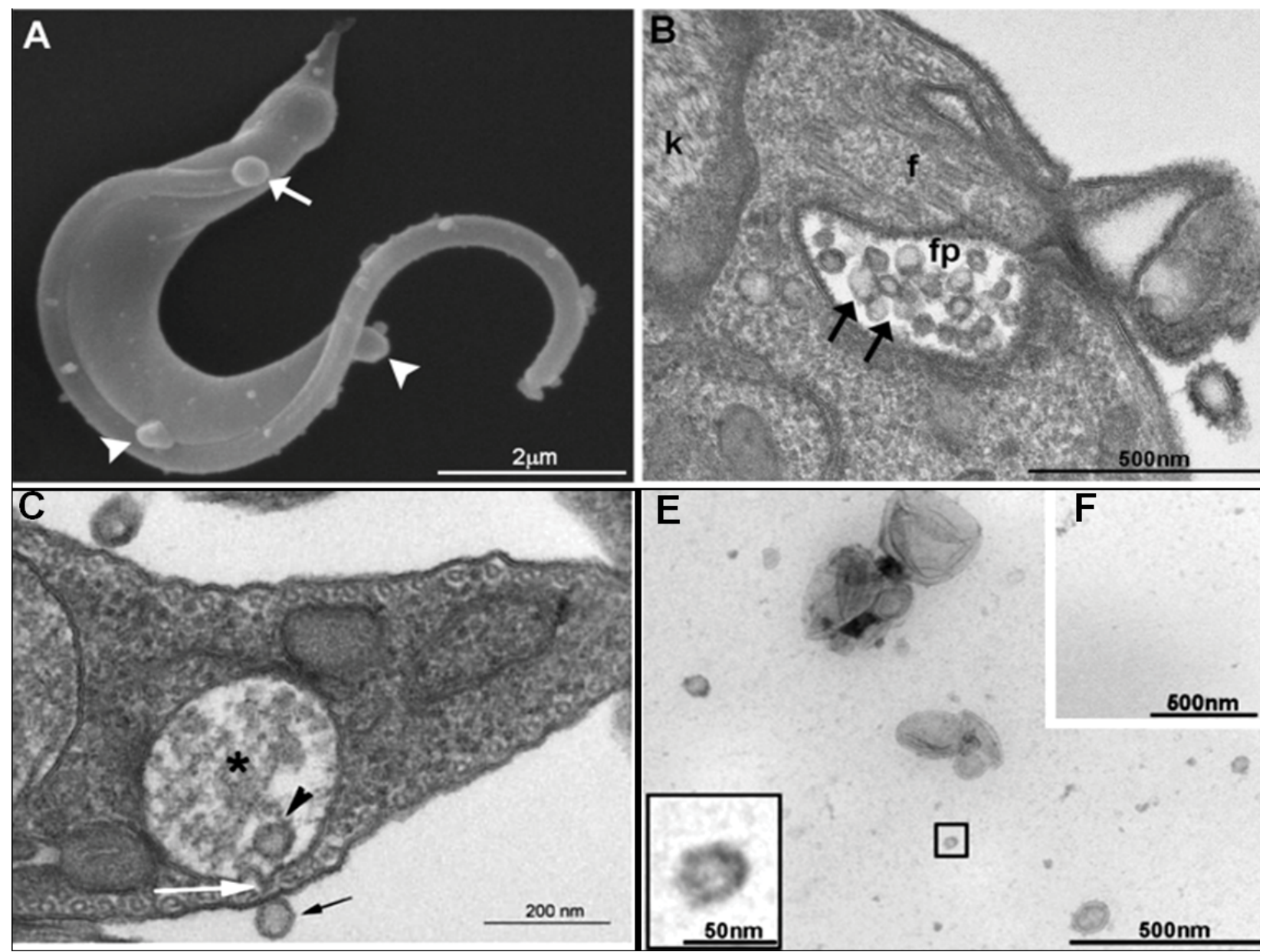

Figure 5: Ultrastructural characterization and size distribution of a vesicle fraction obtained from trypomastigotes (A) SEM of trypomastigote releasing vesicles in different regions of the cell body (white arrowheads) and the flagellum (white arrow); (B) TEM image of small vesicles inside the flagellar pocket of a trypomastigote form (black arrows). Note that vesicles present different sizes; (C) TEM image of reservosome vesicles. Note that the two vesicles in e (arrowhead and black arrow) present the same size and that the membrane of the reservosome (asterisk) is in close contact with the cell membrane (white arrow); (D) Negative staining of the vesicle fraction demonstrating some heterogeneity in size; (E) No vesicles were observed in the supernatant of the vesicle fraction. After Neves, et al. [47].

colleagues [55] showed that culture derived trypomastigotes and metacyclic trypomastigotes induce different levels of MV release from THP-1 cells. They then used Fluorescence Resonance Energy Transfer (FRET) microscopy to show that THP-1-derived microvesicles can fuse with those produced by the parasite. Sera from patients with chronic Chagas disease in the indeterminate or cardiac phase also differentially recognized antigens in THP-1-derived microvesicles resulting only from interaction with infective stages demonstrating a possible modulation of the immune system.

Bayer-Santos, et al. [56] recently analyzed vesicles isolated from $T$. cruzi epimastigotes and metacyclic trypomastigotes from the Dm28c strain. The results showed that the vesicles were composed of a wide variety of RNA molecules including rRNA, mRNAs, and small RNAs. Importantly, the authors also observed some differences in composition between vesicles isolated from epimastigotes and those from metacyclic trypomastigotes. For instance, while tRNA comprised $35 \%$ of all small RNAs from epimastigotes, this percentage was only $6.5 \%$ in the case of bloodstream trypomastigotes.
Nogueira, et al. [57] isolated vesicles from trypomastigotes of four different $T$. cruzi strains $(Y$, Colombiana, CL-14 and YuYu) and found that YuYu parasites released more vesicles than the other strains. These vesicles were also richer in proteins while those from the $Y$ strain showed more alpha-galactosyl-containing glycoconjugates. Differences in the ability of the vesicles from different strains to induce innate and chronic immune response were observed.

The following components have been detected in T. cruzi exosomes: TGF- $\beta$, GP63, GP82, GP85/trans-sialidase family, GP90, TCSMP, $\alpha$-galactosyl glycoconjugates, cruzipain, SAP proteins, TcPIWI-tryp, FCaBP, and Cyclophilin A $[37,58]$.

Certainly further studies are necessary to clarify the role played by these small RNAs including the life cycle and behavior of the different strains of $T$. cruzi. Recent studies have revealed that rRNA-derived small RNAs are processed by special enzymes and might play important roles in heterochromatin assembly and transcription regulators $[59,60]$. 


\section{Conclusions}

Based on the available data we speculate that the microvesicles play some role in the biology of $T$. cruzi as well as on the pathogenesis of Chagas disease. Here, inflammatory processes and host cell dead occur as a consequence of parasites, factors released by the parasites, and molecules produced by different host cells, which are part of the immunological response. This hypothesis is based on the following: (a) Electron microscopy images show release of microvesicles by trypomastigotes as well by intracellular amastigotes, (b) The presence of some key proteins in the microvesicles such as transialidase, mucins, etc., and (c) The presence of RNA molecules that contain genetic information that may interfere with transcription processes.

\section{Acknowledgments}

The work carried out in the authors laboratory has been supported by Conselho Nacional de Desenvolvimento Científico e Tecnológico-CNPq, Financiadora de Estudos e Projetos-Finep, Fundação de Coordenação de Aperfeiçoamento de Pessoal de Nível Superior-CAPES, and Fundação Carlos Chagas Filho de Amparo à Pesquisa do Estado do Rio de Janeiro-FAPERJ.

\section{References}

1. Bruce Alberts, Alexander Johnson, Julian Lewis, Martin Raff, Keith Roberts, et al. (2002) Molecular Biology of the Cell. ( $4^{\text {th }}$ edn), Garland Science, New York.

2. Pan BT, Johnstone RM (1983) Fate of the transferrin receptor during maturation of sheep reticulocytes in vitro: selective externalization of the receptor. Cell 33: 967-978.

3. Pan BT, Teng K, Wu C, Adam M, Johnstone RM (1985) Electron microscopic evidence for externalization of the transferrin receptor in vesicular form in sheep reticulocytes. J Cell Biol 101: 942-948.

4. Mathivanan S, Ji H, Simpson RJ (2010) Exosomes: extracellular organelles important in intercellular communication. J Proteomics 73: 1907-1920.

5. Cocucci E, Racchetti G, Meldolesi J (2009) Shedding microvesicles: artefact no more. Trends Cell Biol 19: 43-51.

6. Gould SJ, Raposo G (2013) As we wait: coping with an imperfect nomenclature for extracellular vesicles. J Extracell Vesicles 2.

7. Cocucci E, Meldolesi J (2015) Ectosomes and exosomes: shedding the confusion between extracellular vesicles. Trends Cell Biol 25: 364-372.

8. McGough IJ, Vincent JP (2016) Exosomes in developmental signalling. Development 143: 2482-2493.

9. Ogawa Y, Kanai-Azuma M, Akimoto Y, Kawakami H, Yanoshita R (2008) Exosome-like vesicles with dipeptidyl peptidase IV in human saliva. Biol Pharm Bull 31: 1059-1062.

10. Ogawa Y, Kanai-Azuma M, Akimoto Y, Kawakami H, Yanoshita R (2008) Exosome-like vesicles in Gloydius blomhoffii blomhoffii venom. Toxicon 51: 984-993.

11. Van Niel G, Porto-Carreiro I, Simões S, Raposo G (2006) Exosomes: a common pathway for a specialized function. $J$ Biochem 140: 13-21.
12. Schorey JS, Bhatnagar S (2008) Exosome function: from tumor immunology to pathogen biology. Traffic 9: 871-881.

13. Denzer K, Keijmeer MJ, Heijnen HF, Stoorvogel W, Geuze HJ (2000) Exosome: from internal vesicle of the multivesicular body to intercellular signaling device. J Cell Sci 113: 3365-3374.

14. Lakkaraju A, Rodriguez-Boulan E (2008) Itinerant exosomes: emerging roles in cell and tissue polarity. Trends Cell Biol 18: 199-209.

15. Pfeffer SR (2010) Two Rabs for exosome release. Nat Cell Biol 12: 3-4.

16. Zheng Y, Campbell EC, Lucocq J, Riches A, Powis SJ (2013) Monitoring the Rab27 associated exosome pathway using nanoparticle tracking analysis. Exp Cell Res 319: 1706-1713.

17. Fukuda M (2013) Rab27 effectors, pleiotropic regulators in secretory pathways. Traffic 14: 949-963.

18. Valadi H, Ekstrom K, Bossios A, Sjostrand M, Lee JL, et al. (2007) Exosome-mediated transfer of mRNAs and microRNAs is a novel mechanism of genetic exchange between cells. Nat Cell Biol 9: 654-659.

19. Yu X, Odenthal M, Fries JW (2013) Exosomes as miRNA Carriers: Formation-Function-Future. Int J Mol Sci 17: E2028.

20. Raposo G, Nijman HW, Stoorvogel W, Liejendekker R, Harding CV, et al. (1996) B lymphocytes secrete antigen-presenting vesicles. J Exp Med 183: 1161-1172.

21. Morelli AE, Larregina A, Shufesky WJ, Sullivan MG, Stolz DB, et al. (2004) Endocytosis, intracellular sorting, and processing of exosomes by dendritic cells. Blood 104: 32573266.

22. Record M, Subra C, Silvente-Poirot S, Poirot M (2011) Exosomes as intracellular signalosomes and pharmacological effectors. Biochem Pharmacol 81: 1171-1182.

23. Izquierdo-Useros N, Puertas MC, Borras FE, Blanco J, Martinez-Picado $\mathrm{J}$ (2011) Exosomes and retrovirus: the chicken or the egg? Cell Microbiol 13: 10-17.

24. Fevrier B, Vilette D, Laude H, Raposo G (2005) Exosomes: a bubble ride for prions? Traffic 6: 10-17.

25. Février B, Raposo G (2004) Exosomes: endosomal-derived vesicles shipping extracellular messages. Curr Opin Cell Biol 16: 415-421.

26. Pelchen-Matthews A, Raposo G, Marsh M (2004) Endosomes, exosomes and Trojan viruses. Trends Microbiol 12: 310-316.

27. Meckes DG Jr, Shair KH, Marquitz AR, Kung CP, Edwards $\mathrm{RH}$, et al. (2010) Human tumor virus utilizes exosomes for intercellular communication. Proc Natl Acad Sci U S A 107: 20370-20375

28. Silverman JM, Reiner NE (2011) Exosomes and other microvesicles in infection biology: organelles with unanticipated phenotypes. Cell Microbiol 13: 1-9.

29. Bukong TN, Momen-Heravi F, Kodys K, Bala S, Szabo G (2014) Exosomes from hepatitis $C$ infected patients transmit HCV infection and contain replication competent viral RNA in complex with Ago2-miR122-HSP90. PLoS Pathog 10: e10004424.

30. Silverman JM, Clos J, de Oliveira CC, Shirvani O, Fang $Y$, et al. (2010) An exosome-based secretion pathway is responsible for protein export from Leishmania and communication with macrophages. J Cell Sci 123: 842-852. 
31. Atayde VD, Aslan H, Townsend S, Hassani K, Kamhawi S, et al. (2015) Exosome Secretion by the Parasitic Protozoan Leishmania within the Sand Fly Midgut. Cell Reports 13: $957-$ 967.

32. Eliaz D, Kannan S, Shaked H, Arvatz G, Tkacz ID, et al. (2017) Exosome secretion affects social motility in Trypanosoma brucei. PLOS Pathol 13: e1006245.

33. Geiger A, Hirtz C, Bécue T, Bellard E, Centeno D, et al. (2010) Exocytosis and protein secretion in Trypanosoma. BMC Microbiol 10: 20.

34. Regev-Rudzki N, Wilson DW, Carvalho TG, Sisquella X, Coleman BM, et al. (2013) Cell-cell communication between malaria-infected red blood cells via exosome-like vesicles. Cell 153: 1120-1133.

35. Atayde VD, Hassani K, da Silva Lira Filho A, Borges AR, Adhikari A, et al. (2016) Leishmania exosomes and other virulence factors: Impact on innate immune response and macrophage functions. Cell Immunol 309: 7-18.

36. Marcilla A, Martin-Javlar L, Trellis M, Menezes-Neto A, Osuna $A$, et al. (2014) Extracellular vesicles in parasitic diseases. J Extracel Vesicles 3: 25040.

37. Anthony J Szempruch, Lauren Dennison, Rudo Kieft, John M Harrington, Stephen L Hajduk (2016) Sending a message: extracellular vesicles of pathogenic protozoan parasites. Nature Reviews Microbiology 14: 669-675.

38. Rassi A Jr, Rassi A, Marcondes de Rezende J (2012) American trypanosomiasis (Chagas disease). Infect Dis Clin North Am 26: 275-291.

39. Castro DP, Seabra SH, Garcia ES, de Souza W, Azambuja P (2007) Trypanosoma cruzi: Ultrastructural studies of adhesion, lysis and biofilm formation by Serratia marcescens. Exp Parasitol 117: 201-207.

40. Alves CR, Albuquerque-Cunha JM, Mello CB, Garcia ES, Nogueira NF, et al. (2007) Trypanosoma cruzi: Attachment to perimicrovillar membrane glycoproteins of Rhodnius prolixus. Exp Parasitol 116: 44-52.

41. Rueda K, Trujillo JE, Carranza JC, Vallejo GA (2014) Oral transmission of Trypanosoma cruzi : a new epidemiological scenario for Chagas' disease in Colombia and other South American countries. Biomedica 34: 631-641.

42. Tecia M Ulisses de Carvalho, Wanderley de Souza (1989) Early events related with the behavior of Trypanosoma cruzi within an endocytic vacuole in mouse peritoneal macrophages. Cell Structure and Function 14: 383-392.

43. Andrews NW, Abrams CK, Slatin SL, Griffiths G (1990) A T. cruzi-secreted protein immunologically related to the complement component C9: evidence for membrane pore-forming activity at low pH. Cell 61: 1277-1287.

44. Schmunis GA, Szarfman A, de Souza W, Langembach T (1980) Trypanosoma cruzi: antibody-induced mobility of surface antigens. Experimental Parasitology 50: 90-102.

45. Gonçalves MF, Umezawa ES, Katzin AM, de Souza W, Alves MJ, et al. (1991) Trypanosoma cruzi: shedding of surface antigens as membrane vesicles. Exp Parasitol 72: 43-53.

46. Trocoli Torrecilhas AC, Tonelli RR, Pavanelli WR, da Silva JS, Schumacher RI, et al. (2009) Trypanosoma cruzi: parasite shed vesicles increase heart parasitism and generate an acute inflammatory response. Microbes Infect 11: 29-39.

47. Neves RF, Fernandes AC, Meyer-Fernandes JR, Souto-Padrón T (2014) Trypanosoma cruzi-secreted vesicles have acid and alkaline phosphatase activities capable of increasing parasite adhesion and infection. Parasitol Res 113: 2961-2972.
48. Garcia-Silva MR, Frugier M, Tosar JP, Correa-Domingues A, Ronalte-Alves L, et al. (2010) A population of t-RNA-derived small RNAs is actively produced in Trypanosoma cruzi and recruited to a specific cytoplasmic granules. Mol Biochem Parasitol 171: 64-73.

49. Garcia-Silva MR, Tosar JP, Frugier M, Pantano S, Bonilla $B$, et al. (2010) Cloning, characterization and subcellular localization of a Trypanosoma cruzi argonaute protein defining a new subfamily distinctive of trypanosomatids. Gene 466: $26-35$

50. de Souza W, Sant'anna C, Cunha e Silva NL (2009) Electron microscopy and cytochemistry analysis of the endocytic pathway in pathogenic protozoa. Prog Histochem Cytochem 44: 67-124.

51. Garcia-Silva MR, Cura das Neves RF, Cabrera-Cabrera F, Sanguinetti J, Medeiros LC, et al. (2014) Extracellular vesicles shed by Trypanosoma cruzi are linked to small RNA pathways, life cycle regulation and susceptibility to infection of mammalian cells. Parasitol Res 113: 285-304.

52. Bayer-Santos E, Aguliar-Bonavides C, Rodrigues SP, Cordero EM, Marques AF, et al. (2013) Proteomic analysis of Trypanosoma cruzi secretome. characterization of two populations of extracellular vesicles and soluble proteins. $J$ Proteom Res 12: 883-897.

53. Fernandez-Calero T, Garcia-Silva R, Pena A, Robello C, Persson $\mathrm{H}$, et al. (2015) Profiling of small RNA cargo of extracellular vesicles shed by Trypanosoma cruzi reveals a specific-extracellular signature. Mol Biochem Parasitol 199: 19-28.

54. Cestari I, Ansa-Addo E, Deolindo P, Inai JM, Ramirez MI (2012) Trypanosoma cruzi immune evasion mediated by host cell derived microvesicles. J Immunol 188: 1942-1952.

55. Ramirez MI, Deolindo P, de Messias-Reason IJ, Arigi EA, Choi $\mathrm{H}$, et al. (2017) Dynamic flux of microvesicles modulate parasite-host cell interaction of Trypanosoma cruzi in eukaryotic cells. Cell Microbiol 19.

56. Bayer-Santos E, Lima FB, Ruiz JC, Almeida IC, da Silveira JF (2014) Characterization of the small RNA content of Trypanosma cruzi extacellular vesicles. Mol Biochem Parasitol 193: $71-74$

57. Nogueira PM, Ribeiro K, Silveira AC, Campos JH, Martins Filho CA, et al. (2015) Vesicles from different Trypanosoma cruzi strains tigger differential innate and chronic immune response. J Extracel Vesicles 4: 28734.

58. Borges BC, Uehara IA, Dias LO, Brígido PC, da Silva CV, et al. (2016) Mechanism of infectivity and evasion derived from microvesicles cargo produced by Trypanosoma cruzi. Front Cell Infect Microbiol 6: 161.

59. Li ZH, De Gaudenzi JG, Alvarez VE, Mendiondo N, Wang $\mathrm{H}$, et al. (2012) A 43-nucleotide U-rich element in 3'-untranslated region of large number of Trypanosoma cruzi transcripts is important for mRNA abundance in intracellular amastigotes. J Biol Chem 287: 19058-19069.

60. Cam HP, Sugiyama T, Chen ES, Chen X, FisGeral PC, et al. (2005) Comprehensive analysis of heterochromatin- and RNAi-mediated epigenetic control of the fission yeast genome. Nat Genet 37: 809-819.

61. Teixeira DE, Benchimol M, Crepaldi PH, de Souza W (2012) Interactive multimedia to teach the life cycle of Trypanosoma cruzi, the causative agent of Chagas disease. PLoS Negl Trop Dis 6: e1749. 